\section{Buch- besprechungen}

Gola, Peter: Datenschutz im Call Center, 2. Auflage 2006, Datakontext, ISBN: 3-89577-411-1

Gelungen mit der für ihn charakteristischen knappen Ausführlichkeit führt Gola in die datenschutzrechtlichen Fragen des Call Centers ein. Dem Leser, der mehr wissen möchte, weil es ihn interessiert, werden genug Literaturhinweise geboten.

Nach einem kurzen Vorspann geht es gleich „,zur Sache“, indem der Autor die spezifischen Datenverarbeitungen des Call Centers benennt. Zunächst erklärt Gola die Überwachung der Leistung und des Verhaltens von Arbeitnehmern. Zweitens spricht er Fragen der datenschutzrechtlich zulässigen Nutzung und Weitergabe der Kundentelefonnummer und weiterer Kundendaten sowie nach der zulässigen Erhebung von Interessentendaten an. Und drittens geht er auf die Besonderheiten ein, die sich aus der Weitergabe von Daten der Arbeitnehmer des Call Centers an Auftraggeber ergeben; insbesondere auf das Auftragsdatenverarbeitungsprivileg des $\S 11$ BDSG, auf die Weitergabe von Gesundheitsdaten sowie auf die Weitergabe in Drittstaaten (außerhalb der EU und des EWR). Anders als bei papiergebundenen Kontakt sei zu berücksichtigen, dass im Call Center - wie bei VorOrt-Gesprächen - individuelles Verhalten, individuelle Wünsche und Reaktionen benannt und gespeichert werden können.

Als Verstöße identifiziert Gola vor allem das Fehlen der Einwilligung in telefonische Anrufe (auch durch Annahme der RechtmäBigkeit „,ins Blaue hinein“), die unzulässige Erhebung von Adresslisten für das Telefonmarketing sowie die Verschleierung der oder Täuschung über die Identität des anrufenden Unternehmens.

Das allgemeine Persönlichkeitsrecht schützt im Call Center insbesondere das Recht am gesprochenen Wort. Es ist strafrechtlich durch § 201 StGB geschützt. Wer dagegen verstößt, weil er unbefugt ein Telefonat aufzeichnet oder mit Abhörtechnik mithört, macht sich strafbar (Strafrahmen Freiheitsstrafe bis zu drei Jahren oder Geldstrafe). Die wesentlichen Einschränkungen der Qualitäts- und Leistungskontrol- le ergeben sich aus diesen Vorschriften. Daher setzt sich Gola besonders intensiv mit den Grenzen des Mithörens, insbesondere mit der rechtfertigenden Einwilligung des Call Center-Agents und des Kunden auseinander.

Im praktischen Zusammenhang des Call Centers analysiert Gola sodann auf Basis der Rechtsprechung und Literatur präzise die rechtlichen Grenzen der Datenverarbeitung im Call Center und zwar vornehmlich die Verarbeitung der Arbeitnehmerdaten zu Kontroll- und Leistungsbewertungszwecken. Dargestellt werden auch die arbeitsrechtlichen Fragen, wobei zur Entlastung des Werkes ergänzend auf das Standardwerk von Gola und Wronka zum Arbeitnehmerdatenschutz verwiesen wird.

Auch die Verarbeitung von Kundendaten im Call Center wird angesprochen, muss jedoch angesichts des Leitfadencharakters des Werkes kurz gehalten werden. Dem interessierten Leser gibt Gola einige ausgewählte Literaturempfehlungen. Wichtiger für das Call Center ist die korrekte Durchführung einer Auftragsdatenverarbeitung, die in einem gesonderten Kapital erläutert wird.

Das Werk eignet sich hervorragend für den Datenschutzbeauftragten im Call Center.

\section{Philipp Kramer}

Witt, Bernhard C.: Datenschutz an Hochschulen, Ein Praxishandbuch für Deutschland am Beispiel der Universitäten Baden-Württembergs, Ulm, LegArtis, 2004, 160 Seiten, 25.00 Euro

Der Verfasser legt hier die verbesserte Version seiner an der Fakultät für Informatik der Universität Ulm geschriebenen Diplomarbeit (S. 3) vor. Er hat sich sicherlich redlich darum bemüht, die Rechtsprobleme im Hochschulbereich in BadenWürttemberg in den Griff zu bekommen, doch ist ihm dies trotz der vielen Unterstützung, für die er sich einleitend bedankt (S. $3)$, nicht gelungen.

Der Text gefährdet das informationelle Selbstbestimmungsrecht, etwa mit der unzutreffenden Feststellung, dass eine uneingeschränkte Verarbeitung von Daten zulässig sei, wenn eine Reidentifikation nur mit unverhältnismäßigem Aufwand möglich sei (1.4.3) und mit dem verkürzenden Hinweis: „Ein Übermitteln der Daten für ein anderes Forschungsvorhaben ist zulässig, wenn die Daten ausdrücklich zu wissenschaftlichen Zwecken erhoben wurden“ (S. 69).

Der Verfasser versäumt die Erörterung der grundgesetzlichen Aufteilung der Gesetzgebungskompetenzen zwischen Bund und Länder im Abschnitt 1.4.2, Die Geltungsebenen im Datenschutzrecht. Somit lässt er den Leser ohne ausreichende Hilfe im Dickicht der Vorschriften des Datenschutzes. Der Leser erfährt auch nicht, dass die EG-Datenschutzrichtlinie dem Datenschutzgesetzgeber verpflichtende Vorgaben macht. Hierin liegt ein Mangel des Abschnittes 1.4.3, Allgemein geltende Regelungen.

Ärgerlich (Aufzählung nicht abschließend!) sind

das Fehlen eines Stichwortverzeichnisses,

fehlende Fundstellenangaben im „Verzeichnis grundlegender Rechtsbestimmungen" (Anlage 4),

- eine unschlüssige Gliederung (Ausführungen zu universitärer Forschung gehören nicht in Teil 3 des Buches zur Hochschulselbstverwaltung),

unfertige Abbildungen (Ausweislich der Darstellung in der Abb. 9 kommt der Prüfungsbehörde im Ablauf von Prüfungsverfahren keine Funktion zu.),

vierzehnfache (!) wortgleiche Wiederholung von mehr als einer Seite Text im insgesamt vierzig Seiten umfassenden Teil 4 des Buches

- im Glossar fehlen für den Datenschutz wichtige Begriffe wie z.B. Vertraulichkeit, Verfügbarkeit und Integrität; Anonymisieren.

Das Werk hat einen Nutzen. Die an juristische Fachkunde im Bereich des Datenschutzes $\mathrm{zu}$ stellenden Anforderungen werden deutlich. Wer sich vom Handbuch verlässliche Unterstützung für die Alltagsarbeit erhofft, der aber sei dringend vor der Verwendung des Buches gewarnt! Der Verfasser ist an den hohen Anforderungen des Datenschutzrechts gescheitert.

Ingrid Pahlen-Brandt 